\title{
A Dynamical Study of the Eclipsing Nova OY Arae
}

\section{Citation}

Zhao, Ping, and Jeffrey E. McClintock. 1997. "A Dynamical Study of the Eclipsing Nova OY Arae." The Astrophysical Journal 483 (2): 899-906. https://doi.org/10.1086/304283.

\section{Permanent link}

http://nrs.harvard.edu/urn-3:HUL.InstRepos:41399727

\section{Terms of Use}

This article was downloaded from Harvard University's DASH repository, and is made available under the terms and conditions applicable to Other Posted Material, as set forth at http:// nrs.harvard.edu/urn-3:HUL.InstRepos:dash.current.terms-of-use\#LAA

\section{Share Your Story}

The Harvard community has made this article openly available.

Please share how this access benefits you. Submit a story.

Accessibility 
THE Astrophysical Journal, 483:899-906, 1997 July 10

(C) 1997. The American Astronomical Society. All rights reserved. Printed in U.S.A.

\title{
A DYNAMICAL STUDY OF THE ECLIPSING NOVA OY ARAE
}

\author{
Ping ZhaO ${ }^{1}$ and JefFrey E. McClintock ${ }^{1}$ \\ Harvard-Smithsonian Center for Astrophysics, 60 Garden Street, Cambridge, MA 02138 \\ zhao@cfa.harvard.edu; jem@cfa.harvard.edu \\ Received 1996 December 2; accepted 1997 February 10
}

\begin{abstract}
Photometric and spectroscopic observations of the classical nova OY Ara were carried out using the Cerro Tololo Inter-American Observatory telescopes. We found that OY Ara is an eclipsing system with an orbital period of $0.155466 \pm 0.000003$ days. The radial velocity semiamplitude of the $\mathrm{H} \alpha$ emission line is $118 \pm 9 \mathrm{~km} \mathrm{~s}^{-1}$. Assuming the secondary is near the main sequence, the dynamical analysis shows that the primary and the secondary have masses of $M_{1} \approx 0.82 M_{\odot}$ and $M_{2} \approx 0.34 M_{\odot}$, respectively. We also find that the accretion disk of OY Ara is $2.5-6$ mag more luminous than that of the X-ray novae, presumably due to the much higher rate of mass transfer in classical novae.

Subject headings: binaries: eclipsing — novae, cataclysmic variables — stars: individual (OY Ara) — white dwarfs
\end{abstract}

\section{INTRODUCTION}

The classical nova OY Ara was discovered on 1910 April 4 at a photographic magnitude of $m_{\mathrm{pg}}=6.2$, having risen from a preoutburst brightness of $m_{\mathrm{pg}}=17.5$ (Walker 1923). Approximately 5 weeks after the discovery, an unusual secondary maximum was observed. The decay rate was 3 mag in 80 days, which is moderately fast (Duerbeck 1987). A nebular spectrum was observed 92 days after the appearance of the nova with emission lines due to $\mathrm{H}$ I, [O III] $\lambda \lambda 4363,5007$, and $\mathrm{N}$ III $\lambda 4640$ (Cannon 1916).

The only quiescent spectrum we are aware of was obtained in 1986 (Duerbeck \& Seitter 1987). It shows a blue continuum with $V=18.5 \pm 0.5$, strong Balmer emission lines, and possibly $\mathrm{He}$ I $\lambda 6678$ in emission and $\mathrm{He}$ I $\lambda 4471$ in absorption. IRAS observations of the nova $63 \mathrm{yr}$ after outburst yielded strong detections in the $12,25,60$, and $100 \mu \mathrm{m}$ bands; however, it is probable that these detections at the longer wavelengths are due to contamination by Galactic cirrus and at the shorter wavelengths to source confusion, given the low Galactic latitude of the object $(b=-3.9$; Harrison \& Gehrz 1991).

In 1994-1995 we made spectroscopic and photometric observations of OY Ara as part of a search for new X-ray novae (XRNs, also known as soft X-ray transients) among the many cataloged Galactic novae (Duerbeck 1987). XRNs are mass transfer binaries in which the accreting star is most often a black hole and the secondary is a low-mass $\left(M_{\text {opt }} \lesssim\right.$ $1 M_{\odot}$ ) star (van Paradijs \& McClintock 1995). The optical light curves of XRNs and Galactic novae are indistinguishable, and several XRNs are included in Duerbeck's (1987) catalog. In fact, the XRN V404 Cyg was discovered optically as a classical nova in 1938 and was not identified as an XRN until 1989 (Makino et al. 1989). It seems plausible, therefore, that at least a few of the 277 Galactic novae in Duerbeck's catalog are heretofore unidentified XRNs. Consequently, we have been searching among the cataloged Galactic novae for the spectral signatures of an XRN: exceptionally broad and double-peaked Balmer emission

\footnotetext{
${ }^{1}$ Visiting Astronomer at Cerro Tololo Inter-American Observatory (CTIO), which is operated by the Association of Universities for Research in Astronomy (AURA), Inc., under contract with the National Science Foundation.
}

lines and a near absence of higher excitation lines (e.g., Orosz et al. 1994; Marsh, Robinson, \& Wood 1994).

During the period 1993 June to 1995 June, we completed our survey of several dozen classical novae. We obtained spectra for these novae at $\sim 3-4 \AA$ resolution using the Multiple Mirror Telescope on Mount Hopkins, Arizona, for the northern targets and the Cerro Tololo InterAmerican Observatory (CTIO) Blanco $4 \mathrm{~m}$ telescope for the southern targets. For several of the most promising candidates we have attempted to measure the orbital period and the radial velocities of the secondary in order to determine the value of the mass function, which is a firm lower limit on the mass of the primary. In this way we hoped to distinguish between a white dwarf primary $\left(M_{1} \leq 1.4 M_{\odot}\right)$ and a more massive black hole primary. Nova OY Ara is one of the six systems we studied closely.

\section{OBSERVATIONS}

Duerbeck's (1987) finding chart for OY Ara is ambiguous because it shows a tight cluster of stars at the location of the object. Our spectroscopic and photometric observations reported herein clearly identify the optical counterpart to be the object indicated in the finding chart shown in Figure 1, which is an image taken with the CTIO $0.91 \mathrm{~m}$ telescope on 1995 May 22.

Spectroscopic observations of OY Ara were obtained with the Blanco $4 \mathrm{~m}$ telescope and Ritchey-Chrétien spectrograph at CTIO in 1994 and 1995; a list of the observations is given in Table 1. Most of the observations were made in 1995 June using the Loral 3K CCD $(3072 \times 1024$ pixels) detector and the KPGL 3 grating. The sky conditions were clear, the seeing was good, the width of the entrance slit was set at $\approx 1^{\prime \prime}$, and the spectral resolution was $\approx 3 \AA$ throughout the 1995 observations. The slit was rotated occasionally to maintain approximate alignment with the parallactic angle (Filippenko 1982). The exposures of OY Ara were interspersed with exposures of wavelength calibration lamps (He-Ne-Ar). In 1994 the observing conditions were also good and the spectral resolution was $\approx 4 \AA$. In both 1994 and 1995 we observed flux standards and more than 10 comparison stars (G-M dwarfs). Spectral reductions were performed using the DOSLIT package in IRAF. The wavelength calibrations were interpolated dis- 


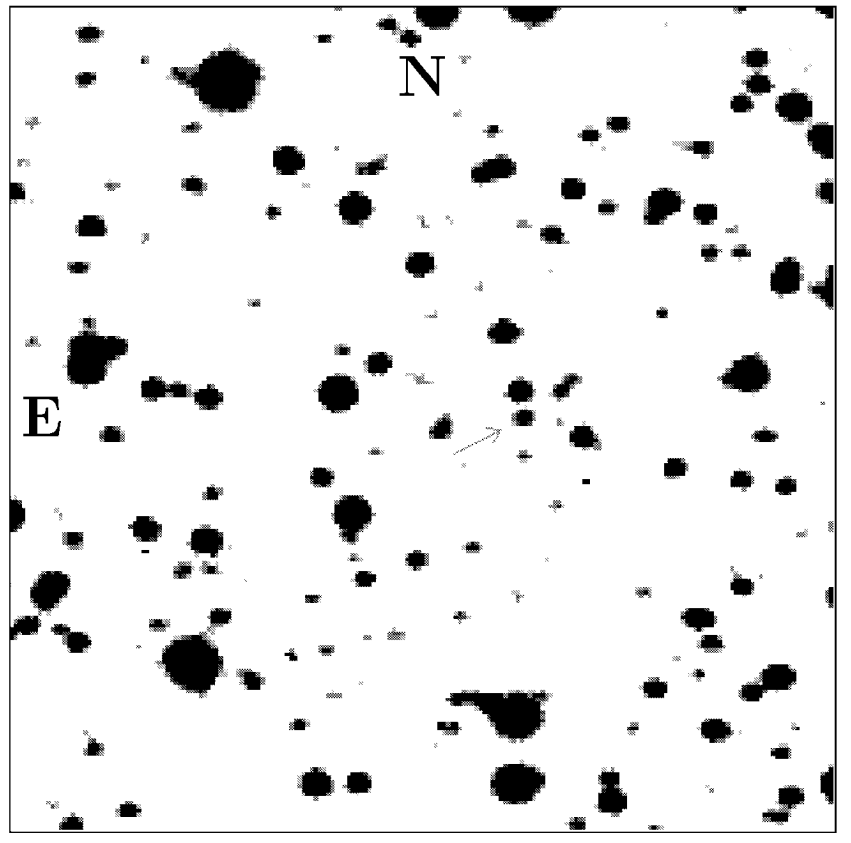

Fig. 1.-Location of Nova OY Ara is indicated by the arrow. This image, which is 1.32 on a side, was taken during eclipse on 1995 May 22 using the $B+V$ filter.

persion solutions, scaled according to the time of an observation relative to the times of the lamp exposures.

Photometric observations were made with the $0.91 \mathrm{~m}$ telescope at CTIO. The observations are listed in Table 2. We used the Tek 2 K CCD $(2048 \times 2048$ pixels $)$ and two broadband filters: a Corning No. 9780 filter (hereafter, $B+V$ ), which has a $2200 \AA$ (FWHM) bandpass centered at $4700 \AA$, and a Schott RG9 filter (hereafter, wide I) which has a $4000 \AA$ (FWHM) bandpass centered at $9000 \AA$. Images were processed to eliminate the electronic bias and correct for pixel-to-pixel sensitivity variations. The relative intensities of OY Ara and selected field stars were determined using the DAOPHOT package in IRAF.

TABLE 1

Spectroscopic ObServations at the CTIO 4 Meter Telescope

\begin{tabular}{|c|c|c|c|c|}
\hline $\begin{array}{l}\text { Date } \\
\text { (UT) }\end{array}$ & $\begin{array}{c}\text { Number of } \\
\text { Spectra }\end{array}$ & $\begin{array}{c}\text { Exposure } \\
\text { Time } \\
\text { (minutes) }\end{array}$ & $\begin{array}{c}\text { Bandwidth } \\
(\AA)\end{array}$ & $\begin{array}{c}\text { Pixel } \\
\text { Size } \\
(\AA)\end{array}$ \\
\hline 1994 July $1 / 2$. & 1 & 20 & $4666-6686$ & 2.0 \\
\hline 1995 June $2 / 3 \ldots \ldots$ & 10 & 20 & $3860-7520$ & 1.2 \\
\hline 1995 June $3 / 4 \ldots \ldots$ & 19 & $10 \& 15$ & $3860-7520$ & 1.2 \\
\hline 1995 June $4 / 5 \ldots \ldots$ & 9 & 10 & $3860-7520$ & 1.2 \\
\hline Total .............. & 39 & $\ldots$ & $\ldots$ & $\ldots$ \\
\hline
\end{tabular}

TABLE 2

Photometric Observations at the CTIO 0.91 Meter Telescope

\begin{tabular}{|c|c|c|c|}
\hline $\begin{array}{l}\text { Date } \\
\text { (UT) }\end{array}$ & $\begin{array}{l}\text { Number of } \\
\text { Images }\end{array}$ & $\begin{array}{l}\text { Exposure Time } \\
\text { (minutes) }\end{array}$ & Filter \\
\hline 1994 July $8 / 9$ & 12 & 15 & Wide I \\
\hline 1994 July $9 / 10$. & 9 & 15 & Wide I \\
\hline 1995 May $22 / 23 \ldots \ldots$ & 72 & $5 \& 10$ & $B+V$ \\
\hline 1995 May $24 / 25 \ldots \ldots$ & 115 & 4 & $B+V$ \\
\hline 1995 May $25 / 26 \ldots \ldots$ & 37 & 4 & $B+V$ \\
\hline 1995 Мay $26 / 27 \ldots \ldots$ & 31 & 4 & $B+V$ \\
\hline Total ................. & 276 & $\ldots$ & $\ldots$ \\
\hline
\end{tabular}

\section{PHOTOMETRIC RESULTS}

Our first observations in 1994 July showed that the light curve of OY Ara has a deep eclipse with a duration of $\$ 30$ minutes. Consequently, in 1995 May we increased the time resolution of our observations (see Table 2). Also, we switched from the wide I filter to the $B+V$ filter because our 1994 photometric data showed that the signal-to-noise ratio is better in the $B+V$ band. We determined the times of the eclipse centers for both 1994 and 1995 by fitting an inverted Lorentzian to the count rate data obtained for each eclipse observation. Using the 1995 data, we made a linear fit of eclipse number versus eclipse time and obtained an orbital period of $0.15540 \pm 0.00011$ days. We obtained a more precise (and unambiguous) period for OY Ara, which is given in Table 3, by including the times of the eclipse observations in 1994. The light curve folded on this period is shown in Figure $2 a$; all of the photometric data obtained with the $B+V$ filter in 1995 May are shown binned into 100 bins per period. The panel displays two full periods. Phase zero is defined to be the center of the eclipse. The epoch of phase zero is given in Table 3. The eclipse is caused, as we

TABLE 3

Photometric and $H \alpha$-Spectroscopic Orbital Parameters

\begin{tabular}{ll}
\hline \hline \multicolumn{1}{c}{ Parameter } & \multicolumn{1}{c}{ Result } \\
\hline$P$ (orbital period, days) $\ldots \ldots \ldots \ldots \ldots$ & $0.155466 \pm 0.000003$ \\
$T_{0}$ (photometric, UT) $\ldots \ldots \ldots \ldots \ldots$ & 1995 May $25.3171 \pm 0.0009$ \\
$T_{0}$ (photometric, heliocentric) $\ldots \ldots$. & HJD $2,449,862.8220 \pm 0.0009$ \\
$\gamma$ velocity $\left(\mathrm{km} \mathrm{s}{ }^{-1}\right) \ldots \ldots \ldots \ldots \ldots \ldots$. & $4 \pm 7$ \\
$K$ velocity $\left(\mathrm{km} \mathrm{s}{ }^{-1}\right) \ldots \ldots \ldots \ldots \ldots \ldots$ & $118 \pm 9$ \\
$\mathrm{H} \alpha$-spectral phase delay $\ldots \ldots \ldots \ldots$ & $+0.138 \pm 0.011$, or $50^{\circ} \pm 4^{\circ}$ \\
\hline
\end{tabular}
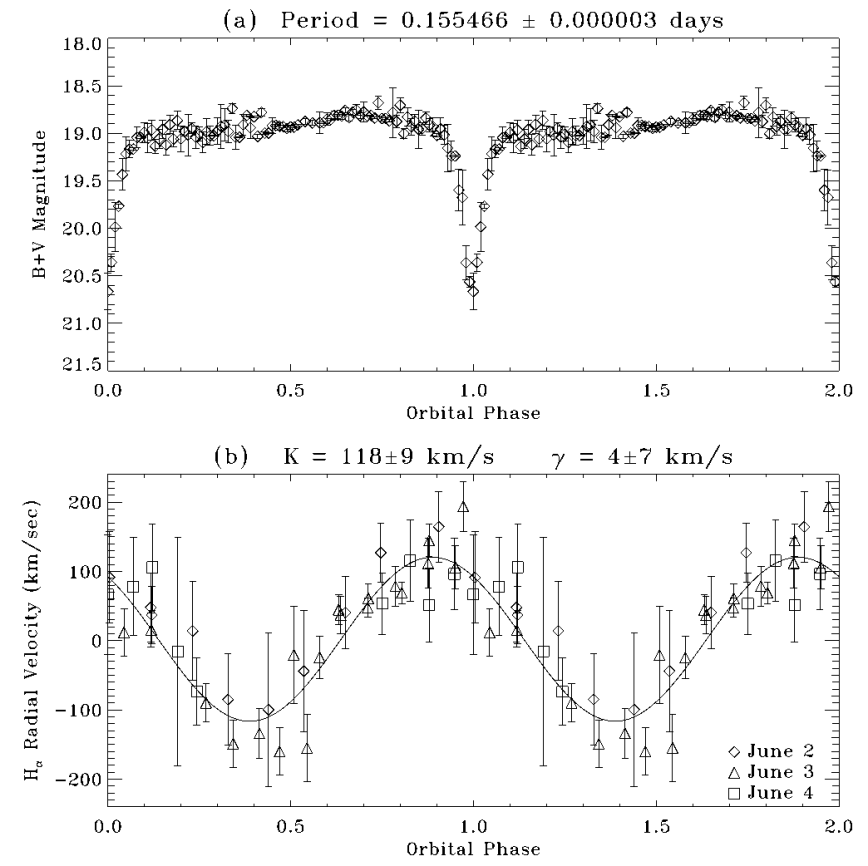

FIG. 2.-Folded light curve and folded $\mathrm{H} \alpha$ radial velocity curve for $\mathrm{OY}$ Ara. (a) All of the photometry data taken with the $B+V$ filter folded on a period of 0.155466 days and binned into 100 phase bins. The zero point of the $B+V$ magnitude scale has been estimated from our spectrophotometric data. (b) The $\mathrm{H} \alpha$ radial velocities folded with the same period and phase. 


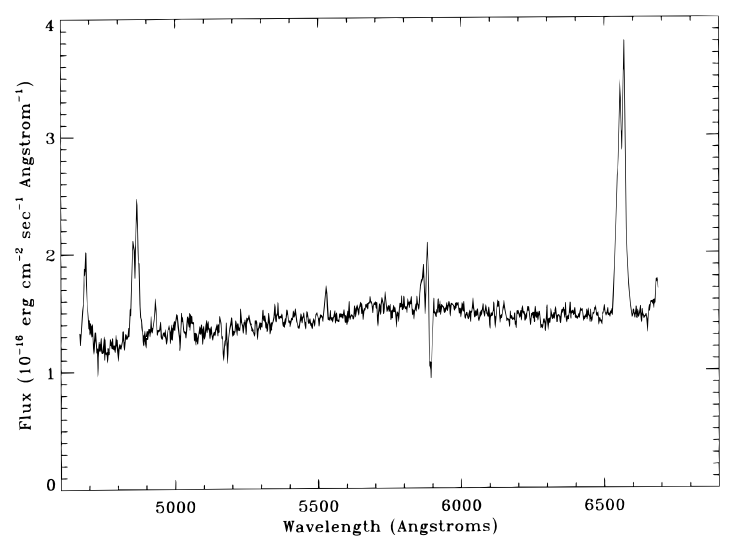

FIG. 3.- Spectrum of OY Ara in quiescence taken 1994 July 1 using the Blanco $4 \mathrm{~m}$ telescope. The Balmer lines are plainly double-peaked.

shall see, by the faint secondary passing in front of the primary and its bright accretion disk.

\section{SPECTROSCOPIC RESULTS}

The single spectrum obtained in 1994 July at photometric phase 0.585 is shown in Figure 3. The most prominent spectral features are the double-peaked Balmer lines and $\mathrm{He}$ I $\lambda 5875$ in emission blended with $\mathrm{NaD}$ in absorption. The equivalent widths of $\mathrm{H} \alpha$ and $\mathrm{H} \beta$ are 48.0 and $22.3 \AA$, and their line widths (FWHM) are 32.1 and $26.7 \AA$, respectively. The values of the equivalent widths and the FWHM were determined by fitting the line profiles with a Gaussian.

A direct sum of all 38 spectra obtained during the 1995 run (June 2-4) is shown with line identifications in Figure 4. The fluxes, equivalent widths, and line widths of the stronger emission lines are summarized in Table 4 . The equivalent widths of the Balmer lines are about the same as in 1994. Disregarding the possible effects of the modest light losses at the spectrograph slit, we note the following differences between the 1995 summed spectrum (Fig. 4) and the 1994 spectrum (Fig. 3): (1) the continuum is slightly bluer in 1995, (2) the Balmer lines are generally single-peaked in 1995 (however, see below) and double-peaked in 1994, and (3) the Balmer lines corrected for the instrumental resolution are narrower in 1995 (the widths of $\mathrm{H} \alpha$ and $\mathrm{H} \beta$ are only about $70 \%-75 \%$ of the widths observed in the single spectrum obtained in 1994). Thus, the spectrum of OY Ara changed markedly on a 1 yr timescale. In addition, the spectrum can change significantly during one orbital cycle. This is shown in Figure 5 where all 38 spectra obtained in 1995 June are binned into 12 phase bins. These 12 spectra are plotted with the same flux and wavelength scales to facilitate comparison; phase zero corresponds to the center of the eclipse. The following two spectral changes occurred during the orbital cycle: (1) the FWHM of the emission lines change during the cycle (from $\sim 20$ to $\sim 31 \AA$ ); they are at their widest and slightly double-peaked near phase 0.4 , around the time of maximum blueshift of the Balmer lines (see below and Fig. $2 b$ ), and (2) the intensities of the emission lines are at maximum near phase 0.75 and decrease to a minimum at phase zero.

We have tried to detect the radial velocity variations of the absorption features from the secondary, using the FXCOR package in IRAF. We used template stars ranging from G8 V to M0 V in the wavelength range 5000-7550 $\AA$ (excluding strong emission and absorption lines such as $\mathrm{H} \alpha$ and $\mathrm{Na} \mathrm{I})$.

For the single OY Ara spectrum from 1994, we did detect significant correlations with several different template stars. Moreover, absorption features, notably Mg I $\lambda \lambda 5167-5184$, can be seen in the 1994 spectrum (Fig. 3). However, this

\section{OY Ara}

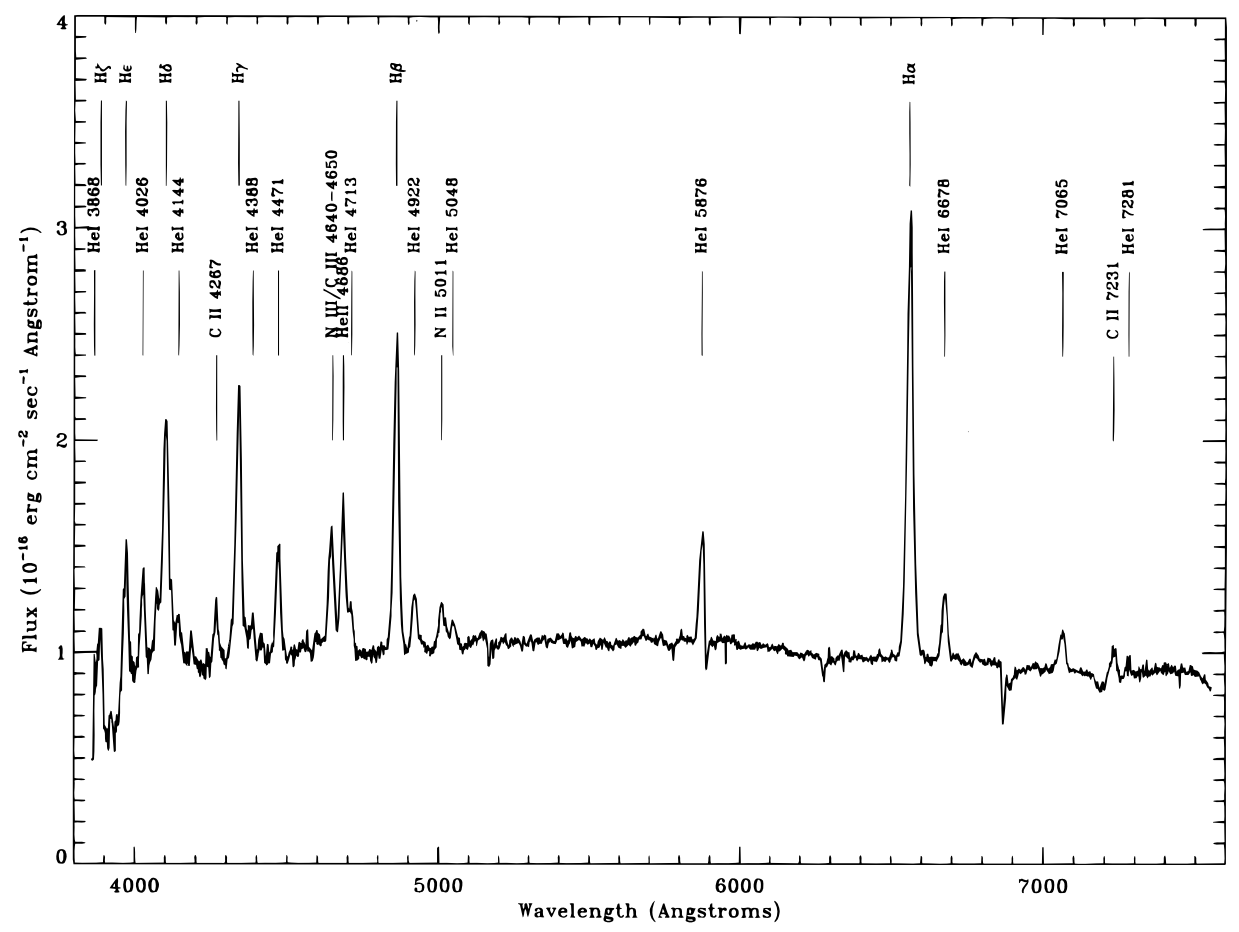

FIG. 4.-The spectrum of Nova OY Ara in quiescence with line identifications. This is a sum of all 38 spectra taken during the nights of 1995 June $2-4$. 
TABLE 4

OY ARA EMISSION LiNeS

\begin{tabular}{|c|c|c|c|c|c|}
\hline Line & $\begin{array}{l}\text { Laboratory } \\
\text { Wavelength } \\
\text { (§) }\end{array}$ & $\begin{array}{c}\text { Line Center } \\
\text { (A) }\end{array}$ & $\begin{array}{c}\text { Flux } \\
\left(10^{-16} \text { ergs }\right. \\
\left.\mathrm{cm}^{-2} \mathrm{~s}^{-1}\right)\end{array}$ & $\begin{array}{l}\text { Equivalent } \\
\text { Width } \\
\text { (§) }\end{array}$ & $\underset{(\AA)}{\mathrm{FWHM}^{\mathrm{a}}}$ \\
\hline $\mathrm{H} \alpha$ & 6563 & 6563.9 & 49.2 & 50.2 & 23.7 \\
\hline $\mathrm{H} \beta \ldots$ & 4861 & 4861.4 & 29.9 & 29.6 & 19.3 \\
\hline $\mathrm{H} \gamma \ldots \ldots \ldots \ldots$ & 4341 & 4340.4 & 24.7 & 23.8 & 19.3 \\
\hline $\mathrm{H} \delta \ldots \ldots \ldots \ldots$ & 4102 & 4101.3 & 15.1 & 12.0 & 16.3 \\
\hline $\mathrm{H} \epsilon \ldots \ldots \ldots \ldots$ & 3970 & 3970.2 & 11.5 & 13.6 & 17.7 \\
\hline $\mathrm{H} \zeta \ldots \ldots \ldots \ldots$ & 3889 & 3889.3 & 3.8 & 4.5 & 13.5 \\
\hline He I $\ldots \ldots \ldots \ldots$ & 7065 & 7064.6 & 4.4 & 4.7 & 23.6 \\
\hline He I $\ldots \ldots \ldots \ldots$ & 6678 & 6678.7 & 6.8 & 7.0 & 20.1 \\
\hline He I $\ldots \ldots \ldots \ldots$ & 5876 & 5875.2 & 9.6 & 9.1 & 17.7 \\
\hline He $\mathbf{I} \ldots \ldots \ldots \ldots$ & 5048 & 5048.9 & 1.1 & 1.1 & 13.8 \\
\hline He I $\ldots \ldots \ldots \ldots$ & 4922 & 4921.5 & 5.4 & 5.3 & 19.5 \\
\hline He г ........... & 4471 & 4471.7 & 9.9 & 10.1 & 17.9 \\
\hline He I $\ldots \ldots \ldots \ldots$ & 4388 & 4387.6 & 2.1 & 2.0 & 13.1 \\
\hline He I $\ldots \ldots \ldots \ldots$ & 4144 & 4143.0 & 3.1 & 3.1 & 18.7 \\
\hline He $\mathbf{I} \ldots \ldots \ldots \ldots$ & 4026 & 4026.7 & 7.8 & 8.5 & 16.9 \\
\hline He II $\ldots \ldots \ldots \ldots$ & 4686 & 4685.3 & 9.0 & 7.9 & 15.4 \\
\hline $\mathrm{C}_{\text {II }} \ldots \ldots \ldots \ldots$ & 7231 & 7231.5 & 4.4 & 5.1 & 27.5 \\
\hline $\mathrm{C}_{\text {II }} \ldots \ldots \ldots \ldots$ & 4267 & 4267.9 & 4.7 & 5.0 & 16.8 \\
\hline $\mathrm{N}_{\mathrm{II}} \ldots \ldots \ldots \ldots$ & 5011 & 5010.3 & 1.8 & 1.6 & 12.7 \\
\hline $\mathrm{N}$ III/C III...... & $4640-4650$ & 4646.4 & 9.6 & 8.9 & 18.3 \\
\hline
\end{tabular}

a A small correction has been applied for an instrumental resolution of $3 \AA$.

feature is an artifact that is the result of contamination by a companion star located only 2".5 north of OY Ara (see Fig. 1). The spatial resolution of the $19941 \mathrm{~K} \times 1 \mathrm{~K} \mathrm{CCD}$ was poor $\left(0.79\right.$ pixel $\left.^{-1}\right)$, and the light from the companion star was blended into the OY Ara spectrum.

In 1995 , the $3 \mathrm{~K} \times 1 \mathrm{~K} \mathrm{CCD}$ had a better spatial resolution $\left(0.5 \mathrm{pixel}^{-1}\right)$, and the companion star was better separated from OY Ara. Consequently, when all 38 individual spectra and 12 phase-binned spectra were crosscorrelated against the template spectra, no absorption features were detected. By adding in a small fraction of the light from a K0 V template star and an M0 V template star to the OY Ara spectra and attempting to "detect" the absorption feature with FXCOR, we found that the continuum contributed by the secondary (assuming its spectral type is in the range $\mathrm{K} 0 \mathrm{~V}$ to $\mathrm{M} 0 \mathrm{~V}$ ) in OY Ara has to be less than $15 \%$ of the total light emitted from the system; otherwise, we would have detected the absorption features. This upper limit agrees with the conclusion obtained by considering the depth of the eclipse (see $\S 6$ ).

The radial velocities of the Balmer lines are modulated at the orbital period and are in phase with each other. We measured the velocities of these lines by fitting a Gaussian profile to the bottom $60 \%$ of each line profile so that the central part of the profile is ignored. These fits determine the line centers, line widths, and levels of the continuum. The radial velocity curve of $\mathrm{H} \alpha$, which is shown in Figure $2 b$, is comprised of all 38 spectra obtained in 1995 June. Making the reasonable assumption that the broad doublepeaked Balmer lines come from the accretion disk (see, e.g., Horne \& Marsh 1986), the $\mathrm{H} \alpha$ radial velocities are expected to reflect the motion of the primary. The fitted velocity semiamplitude, $K=118 \pm 9 \mathrm{~km} \mathrm{~s}^{-1}$, the velocity of the center of mass, $\gamma=4 \pm 7 \mathrm{~km} \mathrm{~s}^{-1}$, and the $\mathrm{H} \alpha$ spectral phase delay (see below) are given in Table 3.

It is evident in Figure 2 that the $\mathrm{H} \alpha$ velocity curve is phase shifted relative to the photometry curve. Phase zero is defined to be mideclipse, which is the time when both the primary and secondary are moving transverse to the line of sight. The $\mathrm{H} \alpha$ radial velocity curve, which is produced by the accretion disk, lags the motion of the primary by $+50^{\circ} \pm 4^{\circ}$ (or $+0.138 \pm 0.011$ in phase). If the accretion disk were centered around the primary, this phase delay should be zero because the phase of the primary is well defined by the system eclipse. This phase delay effect is due to the distortion of the accretion disk, which has been observed for a number of other CVs and for XRNs (Stover 1981; Shafter 1983; Marsh, Horne, \& Shipman 1987; Orosz et al. 1994). Therefore the phase of the velocity curve does not reflect the orbital motion of the primary around the center of mass of the binary.

\section{DYNAMICAL ANALYSIS AND MASS DETERMINATION}

Now we analyze the dynamics of the OY Ara binary system and estimate the masses of its primary and secondary. We first use the mass function of the secondary,

$$
\frac{M_{2}^{3} \sin ^{3} i}{\left(M_{1}+M_{2}\right)^{2}}=\frac{P K_{1}^{3}}{2 \pi G} .
$$

$M_{1}$ and $M_{2}$ are the masses of the primary and the secondary, respectively; $i$ is the angle of inclination; $P$ is the orbital period; $K_{1}$ is the velocity semiamplitude of the primary; and $G$ is the gravitational constant.

From equation (1), we can express $M_{1}$ as a function of $M_{2}$ :

$$
M_{1}=M_{2}\left(\sqrt{\frac{2 \pi G}{P K_{1}^{3}} M_{2} \sin ^{3} i}-1\right) .
$$

Substituting $P$ and $K_{1}{ }^{2}$ from Table 3 into equation (2), we can plot $M_{1}$ as a function of $M_{2}$, as shown in Figure 6 by

\footnotetext{
${ }^{2}$ We assume that $K_{1}$ is the true velocity amplitude of the primary (although the phase of the velocity curve does not reflect the orbital motion of the primary). Evidence that $K_{1}$ is the true amplitude is provided by two independent and consistent determinations of the mass ratio $\left(M_{1} / M_{2}\right)$ of XRN Mus 1991, a system that displays an emission-line phase shift comparable to that of OY Ara (Orosz et al. 1994; Casares et al. 1997).
} 

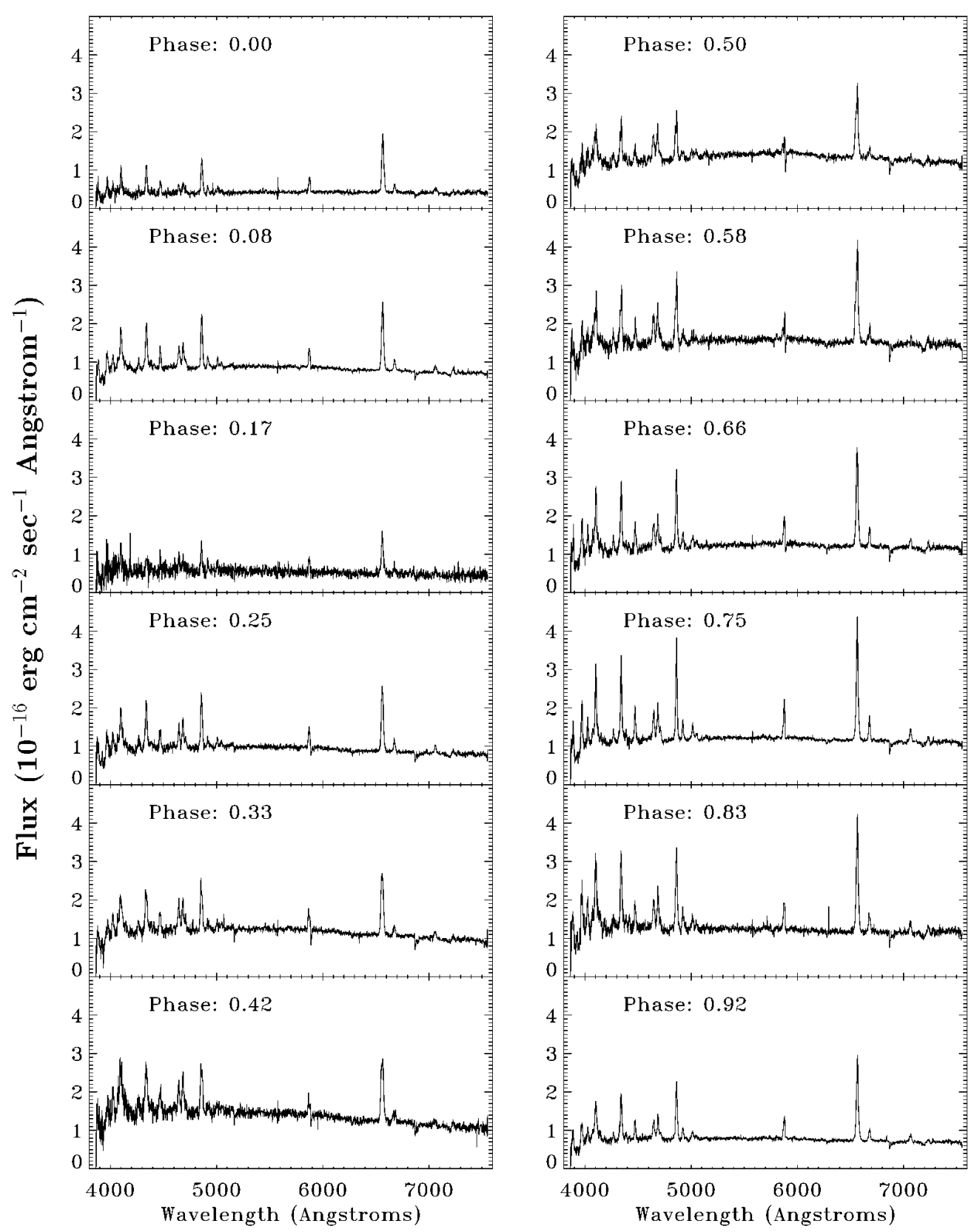

FIG. 5.-Nova OY Ara Spectra binned into 12 phase bins. The faint continuum of the spectrum in phase bin 0.17 is an artifact; only one spectrum contributed to this bin, and it was affected by clouds.

the family of curves that runs from the lower left to the upper right in that figure. Curves are plotted for inclination angles of $90^{\circ}, 75^{\circ} .5,74^{\circ} .3$, and $73^{\circ} .1$. The rationale for these specific angles is given later in this section.

Now we derive an expression for the mass of the secondary. We assume the secondary star fills its Roche lobe, and use the expression for the dimensionless radius of the secondary derived by Eggleton (1983),

$$
\frac{R_{2}}{a}=\frac{0.49 q^{2 / 3}}{0.6 q^{2 / 3}+\ln \left(1+q^{1 / 3}\right)}
$$

where $R_{2}$ is the radius of the secondary (i.e., the Roche lobe), $a$ is the separation between the centers of the stars, and $q$ ( $\left.=M_{2} / M_{1}\right)$ is the mass ratio. We then assume that the secondary is near the main sequence and adopt a power-law mass-radius relation (Patterson 1984):

$$
\frac{R_{2}}{R_{\odot}}=b\left(\frac{M_{2}}{M_{\odot}}\right)^{x}
$$

where $b$ and $x$ are parameters.

Combining equation (4) with Kepler's third law $\left[4 \pi^{2} a^{3}=G\left(M_{1}+M_{2}\right) P^{2}\right]$, the following equation can be derived for the mass of the secondary (Shafter 1984):

$$
\begin{aligned}
\frac{M_{2}}{M_{\odot}} & =\left[\frac{G M_{\odot}}{4 \pi^{2} R_{\odot}^{3}}\left(1+\frac{1}{q}\right) \frac{P^{2}}{b^{3}}\left(\frac{R_{2}}{a}\right)^{3}\right]^{1 /(3 x-1)} \\
& =\left[0.997 \times 10^{-8}\left(1+\frac{1}{q}\right) \frac{P^{2}}{b^{3}}\left(\frac{R_{2}}{a}\right)^{3}\right]^{1 /(3 x-1)},
\end{aligned}
$$




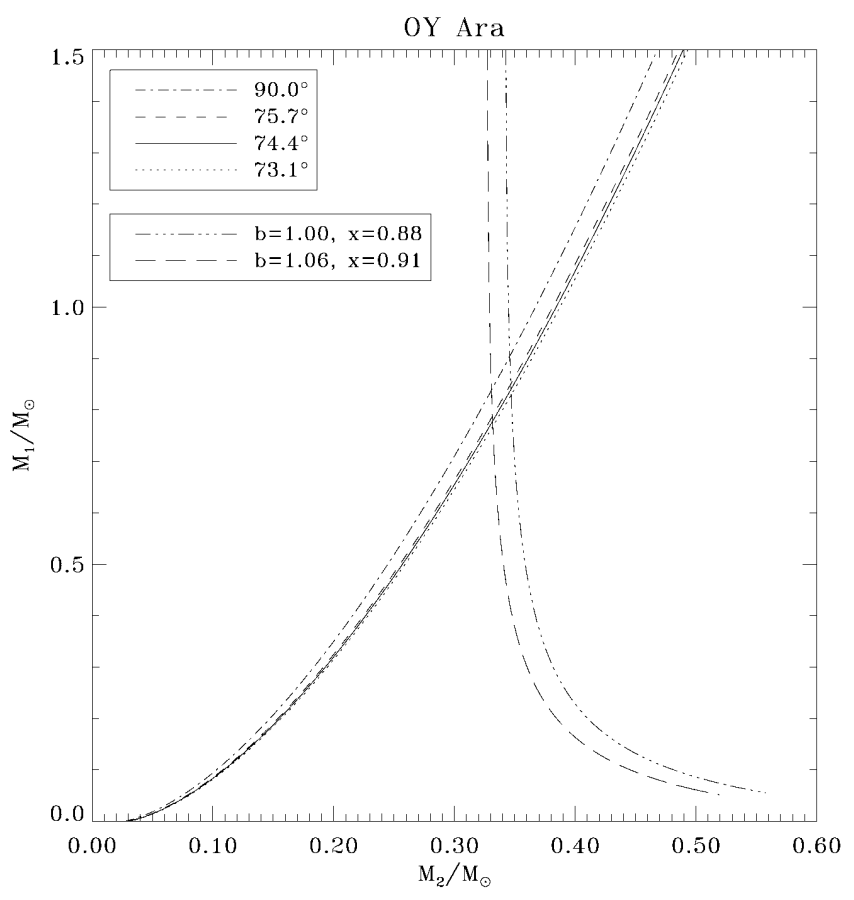

Fig. 6.-Nova OY Ara mass ratio diagram. The primary mass $M_{1}$ is plotted as a function of the secondary mass $M_{2}$. The curves that run from the lower left to the upper right were calculated using the mass function (eq. [2]) with angles of inclination from $i=73.1$ to $i=90^{\circ}$. The two nearly vertical curves assume that the secondary is near the main sequence; the curves correspond to the values of $b$ and $x$ given in the Figure (see text).

where $P$ is the orbital period in seconds, and $\left(R_{2} / a\right)$ is given by equation (3).

Using equation (6) and the relation $M_{1}=M_{2} / q$, we can draw another set of curves in the $M_{1}-M_{2}$ plane. Each such curve is determined by the parameter pair $b$ and $x$. We adopt the values for the lower main sequence given by Patterson (1984): $b=1.0$ and $x=0.88$; and by Echevarria (1983): $b=1.06$ and $x=0.91$. These two curves are plotted in Figure 6. Their intersections with the curves from the mass function give the estimated value of the primary and the secondary:

$$
\begin{aligned}
& M_{1}=0.82 \pm 0.12 M_{\odot}, \\
& M_{2}=0.34 \pm 0.01 M_{\odot},
\end{aligned}
$$

where the errors are mainly due to the uncertainty in the $K_{1}$ velocity. They are also partly due to the uncertainty in the inclination angle. The above mass estimates are based on the assumption that the secondary is near the main

\section{TABLE 5}

Results of Dynamical ANalysis of OY ARA BINARY SYSTEM

\begin{tabular}{lc}
\hline \hline Parameter & Results \\
\hline Mass of primary, $M_{1}\left(M_{\odot}\right) \ldots \ldots \ldots \ldots \ldots$. & $0.82 \pm 0.12$ \\
Mass of secondary, $M_{2}\left(M_{\odot}\right) \ldots \ldots \ldots \ldots \ldots$ & $0.34 \pm 0.01$ \\
Roche radius of primary, $R_{1}\left(R_{\odot}\right) \ldots \ldots \ldots$ & $0.58 \pm 0.04$ \\
Roche radius of secondary, $R_{2}\left(R_{\odot}\right) \ldots \ldots$ & $0.39 \pm 0.01$ \\
System separation, $a\left(R_{\odot}\right) \ldots \ldots \ldots \ldots \ldots \ldots$ & $1.28 \pm 0.04$ \\
System inclination, $i \ldots \ldots \ldots \ldots \ldots \ldots \ldots \ldots$ & $74.3 \pm 1.2$ \\
\hline
\end{tabular}

sequence. If the parameter pair $b$ and $x$ differ from the values given by Patterson (1984) and Echevarria (1983), the estimated masses could be considerably different (Wade 1990). For example, if the secondary were somewhat evolved, both $M_{1}$ and $M_{2}$ could be considerably lower (e.g., for $b=1.2$ and $x=0.91, M_{1} \approx 0.54 M_{\odot}$ and $M_{2} \approx 0.27$ $\left.M_{\odot}\right)$.

Based on its mass, we conclude that the compact primary of the OY Ara system is a white dwarf rather than a neutron star or a black hole.

Using Kepler's third law and the estimated masses, we also obtain the separation between the centers of mass of the primary and the secondary:

$$
a=1.28 \pm 0.04 R_{\odot},
$$

where the error is mainly due to the uncertainty in $M_{1}$.

Using equation (3), we obtain the Roche lobe radius of the secondary $R_{2}$, and, replacing $q$ with $1 / q$ in equation (3), we obtain the Roche lobe radius of the primary $R_{1}$ :

$$
\begin{aligned}
& R_{1}=0.58 \pm 0.04 R_{\odot}, \\
& R_{2}=0.39 \pm 0.01 R_{\odot} .
\end{aligned}
$$

Since OY Ara is an eclipsing system, its inclination angle has to be larger than $\cos ^{-1}\left(R_{2} / a\right) \approx 72^{\circ} .3$.

Now we estimate the inclination angle of the system. For an eclipsing system, the duration of the eclipse $\Delta \varphi$ is a function of $i$ and $q$. For Roche geometry, this function is tabulated (Chanan, Nelson, \& Margon 1978; Horne 1985). Ignoring the distorted shape of the secondary and treating it as a sphere, an analytical formula can be derived that is in very good agreement with the more sophisticated light curve simulations (Horne, Lanning, \& Gomer 1982):

$$
\sin ^{2} i \approx \frac{1-\left(R_{2} / a\right)^{2}}{\cos ^{2} \pi \Delta \varphi},
$$

where $\Delta \varphi$ is the full width at half depth of the eclipse. We fitted the eclipse portion of the light curve (Fig. 2a) with a Lorentzian and obtained $\Delta \varphi=0.048 \pm 0.005$. Substituting this value into formula (12) gives the following value for the orbital inclination angle:

$$
i=74^{\circ} .4 \pm 1.3 .
$$

In Figure 6, we show the mass function curves for $i=$ 74.4 as well as for the upper and lower limits 73.1 and $75^{\circ} .7$. We also show the mass function curve for an inclination of $90^{\circ}$.

As shown in Figure 6, the masses, and therefore $R_{2}$, are not very sensitive to the inclination angle. That is why we used an estimated inclination angle earlier in this section to determine $R_{2}$. Later we used $R_{2}$ to check whether the estimated inclination angle was reasonable.

Table 5 summarizes the dynamical results for the OY Ara binary system.

\section{DISCUSSION}

During eclipse, the secondary passes in front of the primary and its bright accretion disk. The $\sim 2$ mag depth of the eclipse (Fig. $2 a$ ) implies that the secondary must be at least $2 \mathrm{mag}$ dimmer than the accretion disk in the $B+V$ band. This result is in agreement with our nondetection of 
the secondary absorption lines of the secondary, which implies that the secondary contributes less than $15 \%$ of the total light or, equivalently, that the secondary is at least 2 mag dimmer than the accretion disk. The faintness of the secondary is attested to also by the absence of a secondary eclipse when the primary passes in front of the secondary (phase 0.5).

The relative brightness of the secondary and the accretion disk can be estimated as follows. The $3.73 \mathrm{hr}$ orbital period implies that the secondary is very probably a mid-M dwarf with $M_{V} \sim 11.5$ (Patterson 1984, Fig. 4; Ritter \& Kolb 1995). The absolute magnitude of OY Ara in quiescence can be deduced from the amplitude of the outburst and the relationship between $M_{V}(\max )$ and the rate of decline: in this way, Warner (1987, Table) finds that quiescent $M_{V}=4$.6. Thus, the disk probably outshines the secondary by $\sim 7 \mathrm{mag}$ in the $V$ band. Based on our spectrophotometric data, the apparent magnitude of OY Ara in quiescence is $V \approx 18.5$. Given the apparently moderate reddening of the spectrum (see Fig. 4) and $b=-3.9$, we assume $A_{V}=1.0 \mathrm{mag}$. Thus we estimate the distance of $\mathrm{OY}$ Ara to be $\approx 3.8 \mathrm{kpc}$.

The situation is markedly different for quiescent XRNs for two reasons. First, their secondaries are more luminous than the secondary of OY Ara because they are larger $\left(P_{\text {orb }} \geq 5.1 \mathrm{hr}\right.$ ) and hotter (all are M2 or earlier). Second, the accretion disks in XRNs are significantly fainter, a point that we now consider in detail. In Table 6 we compare the absolute magnitude of the accretion disk in OY Ara (entry 1) to the absolute magnitudes of the accretion disks in the six well-studied XRNs with orbital periods $P \lesssim 15 \mathrm{hr}$. The nature of the compact object, the orbital period, and the orbital inclination angle are given in columns (3)-(5), respectively. For the eclipsing system OY Ara, we use an orbital inclination angle of $i=74^{\circ}$, as obtained in $\S 5$. As a measure of the size of the accretion disk in each system, we compute the radius of the Roche lobe of the primary, $R_{1}$ (col. [6]), using Kepler's law, a standard form for the relative radius of the Roche lobe of the primary (Eggleton 1983), and an estimate of the masses of both components. For the six XRNs, we use mass estimates that are given in the references cited in Table 6 to compute $R_{1}$, and for OY Ara we use $R_{1}=0.58 R_{\odot}$, as determined in $\S 5$. Columns (7)-(9) give a distance estimate, the dereddened apparent magnitude of the system, and the fraction of the total light that is contributed by the accretion disk in the $V$ band $\left(f_{V D}\right)$, respectively; all three of these quantities can be obtained from the references cited in Table 6. Note that for OY Ara, $f_{V D}$ is unity (see above), whereas for the XRNs, $f_{V D} \sim 0.5$. The absolute magnitudes of the accretion disks, $M_{V D}$, are given in column (10).

As noted above, the absolute magnitude of the quiescent disk in OY Ara is $M_{V D}=4.6 \mathrm{mag}$. However, this disk is viewed at a high inclination (we assume $i=74^{\circ}$ ) and, consequently, its projected area is small, and its limb darkening is severe. Therefore, if we assume the disk is optically thick (e.g., Williams 1980), the correction from the apparent absolute magnitude of the disk to its absolute magnitude is 1.0 mag, so that the corrected value is $M_{V D}=3.6 \mathrm{mag}$ (see Table 6); this is the predicted absolute magnitude of the disk if viewed at a "nominal" reference inclination of $57^{\circ}$ (Warner 1995).

The accretion disks in quiescent XRNs are less well understood. The disk instability model predicts that the accretion disk of an XRN in quiescence is optically very thick and perhaps as cool as $\sim 2000-3000 \mathrm{~K}$ (Cannizzo 1993, 1996). Such a cool component of emission, which would be difficult to observe, has not been reported. Observations of the disk component of A0620-00 in quiescence, on the hand, can be fitted by a very small $9000 \mathrm{~K}$ blackbody component, which is only $\sim 1 \%$ of the expected area of the accretion disk (McClintock, Horne, \& Remillard 1995); such a component might be due to the action of the accretion stream. An alternative explanation for this hot component is provided by the advection dominated accretion flow model (Narayan, McClintock, \& Yi 1996). In this model, the hot component is due to synchrotron and bremsstrahlung emission from a compact, hot, optically thin corona, which is flowing into the black hole primary. Thus, the nature of the accretion flows in quiescent XRNs is quite uncertain, and therefore we choose not to correct the absolute magnitudes of the XRN (col. [10]). This is of little consequence, because the largest inclination correction would only be $\Delta M_{V D} \approx-0.6 \mathrm{mag}$ (for Cen X-4). We conclude that the accretion disks of six XRNs are quite faint, $M_{V D}=6.0-9.5 \mathrm{mag}$, compared to the accretion disk of OY Ara, $M_{V D}=3.6 \mathrm{mag}$.

The relative faintness of the XRN disks is surprising, since the Roche lobes of their primary stars are much larger than the Roche lobe of the white dwarf in OY Ara (Table 6). Moreover, studies of the $\mathrm{H} \alpha$ profile in XRNs show that the disks themselves are large, extending outward in radius to $\sim 0.5-0.8 R_{1}$ (Orosz et al. 1994; Marsh et al. 1994). Thus, geometrically, the XRN disks are much larger; why then are

TABLE 6

ACCRETION Disk Luminosities For OY ARA AND Six X-RAY Novae

\begin{tabular}{|c|c|c|c|c|c|c|c|c|c|c|}
\hline $\begin{array}{c}\text { Entry } \\
\text { Number } \\
(1)\end{array}$ & $\begin{array}{l}\text { Object } \\
\text { (2) }\end{array}$ & $\begin{array}{l}\text { Primary }^{a} \\
\text { (3) }\end{array}$ & $\begin{array}{c}P \\
(\mathrm{hr}) \\
(4)\end{array}$ & $\begin{array}{c}i \\
(\mathrm{deg}) \\
(5)\end{array}$ & $\begin{array}{c}R_{1} \\
\left(R_{\odot}\right) \\
(6)\end{array}$ & $\begin{array}{c}d \\
(\mathrm{kpc}) \\
(7)\end{array}$ & $\begin{array}{l}V_{0} \\
(8)\end{array}$ & $\begin{array}{c}f_{V D} \\
(9)\end{array}$ & $\begin{array}{c}M_{V D} \\
(10)\end{array}$ & $\begin{array}{l}\text { Ref. } \\
\text { (11) }\end{array}$ \\
\hline $1 \ldots \ldots$ & OY Ara & WD & 3.73 & 74 & 0.58 & 3.8 & 17.5 & 1.0 & $3.6^{\mathrm{b}}$ & 1 \\
\hline $2 \ldots \ldots \ldots$ & GRO J0422 + 32 & BH & 5.08 & 48 & 1.36 & 3.6 & 21.7 & 0.6 & 9.5 & $2-4$ \\
\hline $3 \ldots \ldots$ & $\mathrm{A} 0620-00$ & BH & 7.75 & 55 & 2.25 & 1.2 & 17.2 & 0.4 & 7.8 & 5 \\
\hline $4 \ldots \ldots$ & GS $2000+25$ & $\mathrm{BH}$ & 8.26 & 66 & 2.62 & 2.7 & 19.2 & 0.3 & 8.3 & 5 \\
\hline $5 \ldots$ & GRS $1124-68$ & BH & 10.4 & 60 & 2.54 & 6.5 & 19.4 & 0.5 & 6.0 & 5 \\
\hline $6 \ldots \ldots$ & H1705-25 & $\mathrm{BH}$ & 12.5 & 60 & 3.26 & 8.6 & 20.0 & 0.4 & 6.3 & 5 \\
\hline $7 \ldots \ldots$ & Cen X-4 & NS & 15.1 & 36 & 2.04 & 1.2 & 18.1 & 0.3 & 8.7 & 6 \\
\hline
\end{tabular}

Note.-References: (1) This work; (2) Filippenko, Matheson, \& Ho 1995; (3) Shrader et al. 1994; (4) Garcia et al. 1996; (5) Barret, McClintock, \& Grindlay 1996, and references therein; (6) McClintock \& Remillard 1990.

${ }^{\mathrm{a}} \mathrm{WD}=$ white dwarf; $\mathrm{BH}=$ black hole; $\mathrm{NS}=$ neutron star.

${ }^{\mathrm{b}}$ Corrected for high inclination; the uncorrected value is $M_{V D}=4.6$ (see text). 
these disks typically $\sim 4$ mag fainter than OY Ara? The primary reason is the high rate of mass transfer in quiescent novae, and the modest rates of mass transfer in XRNs. The rates are exceptionally high for a nova before and after an outburst: $\dot{M}_{d} \sim 10^{-8} M_{\odot} \mathrm{yr}^{-1}$ (Patterson 1984; Truran \& Livio 1986). For XRNs in quiescence, however, the rates are $\sim 10^{2}-10^{3}$ times lower (McClintock et al. 1995).
We owe a great deal of thanks to the staff at CTIO. We thank M. Garcia and the referee for their comments on the manuscript. This work was supported in part by NASA grant NAG8-792. Additional support was also provided to J. E. M. by the Smithsonian Institution Scholarly Studies Program.
Barret, D., McClintock, J. E., \& Grindlay, J. E. 1996, ApJ, 473, 963

Cannizzo, J. K. 1993, in Accretion Disks in Compact Stellar Systems, ed.

J. C. Wheeler (Singapore: World Scientific), 6 1996, private communication

Cannon, A. J. 1916, Ann. Astron. Obs. Harvard Coll., 76, 19

Casares, J., Martin, E. L., Charles, P. A., Molaro, P., \& Rebolo, R. 1997, New Astron., 1(4), 299

Chanan, G. A., Nelson, J. E., \& Margon, B. 1978, ApJ, 226, 963

Duerbeck, H. W. 1987, A Reference Catalogue and Atlas of Galactic Novae (Dordrecht: Reidel)

Duerbeck, H. W., \& Seitter, W. C. 1987, Ap\&SS, 131, 467

Echevarria, J. 1983, Rev. Mexicana Astron. Astrofis., 8, 109

Eggleton, P. P. 1983, ApJ, 268, 368

Filippenko, A. V. 1982, PASP, 94, 715

Filippenko, A. V., Matheson, T., \& Ho, L. C. 1995, ApJ, 455, 614

Garcia, M. R., Callanan, P. J., McClintock, J. E., \& Zhao, P. 1996, ApJ, 460, 932

Harrison, T. E., \& Gehrz, R. D. 1991, AJ, 101, 587

Horne, K. 1985, MNRAS, 213, 129

Horne, K., Lanning, H. H., \& Gomer, R. H. 1982, ApJ, 252, 681

Horne, K., \& Marsh, T. R. 1986, MNRAS, 218, 761

Makino, F., et al. 1989, IAU Circ. 4782

Marsh, T. R., Horne, K., \& Shipman, H. L. 1987, MNRAS, 225, 551

Marsh, T. R., Robinson, E. L., \& Wood, J. H. 1994, MNRAS, 266, 137

McClintock, J. E., Horne, K., \& Remillard, R. A. 1995, ApJ, 442, 358

\section{REFERENCES}

McClintock, J. E., \& Remillard, R. A. 1990, ApJ, 350, 386

Narayan, R., McClintock, J. E., \& Yi, I. 1996, ApJ, 457, 821

Orosz, J. A., Bailyn, C. D., Remillard, R. A., McClintock, J. E., \& Foltz, C. B. 1994, ApJ, 436, 848

Patterson, J. 1984, ApJS, 54, 443

Ritter, H., \& Kolb, U. 1995, in X-ray Binaries, ed. W. H. G. Lewin, J. van Paradijs, \& E. P. J. van den Heuvel (Cambridge: Cambridge Univ. Press), 578

Shafter, A. W. 1983, Ph.D. thesis, UCLA

. 1984, AJ, 89, 1555

Shrader, C. R., Wagner, R. M., Hjellming, R. M., Han, X. H., \& Starrfield, S. G. 1994, ApJ, 434, 698

Stover, R. J. 1981, ApJ, 249, 673

Truran, J. W., \& Livio, M. 1986, ApJ, 308, 721

van Paradijs, J., \& McClintock, J. E. 1995, in X-ray Binaries, ed. W. H. G. Lewin, J. van Paradijs, \& E. P. J. van den Heuvel (Cambridge: Cambridge Univ. Press), 58

Wade, R. A. 1990, Accretion-Powered Compact Binaries, ed. C. W. Mauche (Cambridge: Cambridge Univ. Press), 181

Walker, A. D. 1923, Ann. Astron. Obs. Harvard Coll., 84, 189

Warner, B. 1987, MNRAS, 227, 23

. 1995, Cataclysmic Variable Stars (Cambridge: Cambridge Univ. Press), 64

Williams, R. E. 1980, ApJ, 235, 939 